\title{
On Holomorphic $L^{2}$ Functions on Coverings of Strongly Pseudoconvex Manifolds
}

By

Alexander BRUDNYI*

\begin{abstract}
In this paper we answer an important question posed in the paper [GHS] by Gromov, Henkin and Shubin on existence of sufficiently many holomorphic $L^{2}$ functions on arbitrary coverings of strongly pseudoconvex manifolds.
\end{abstract}

\section{$\S 1 . \quad$ Introduction}

1.1. Let $M \subset \subset N$ be a domain with smooth boundary $b M$ in an $n$-dimensional complex manifold $N$, specifically,

$$
M=\{z \in N: \rho(z)<0\}
$$

where $\rho$ is a real-valued function of class $C^{2}(\Omega)$ in a neighbourhood $\Omega$ of the compact set $\bar{M}:=M \cup b M$ such that

$$
d \rho(z) \neq 0 \text { for all } z \in b M .
$$

Let $z_{1}, \ldots, z_{n}$ be complex local coordinates in $N$ near $z \in b M$. Then the tangent space $T_{z} N$ at $z$ is identified with $\mathbb{C}^{n}$. By $T_{z}^{c}(b M) \subset T_{z} N$ we denote the complex tangent space to $b M$ at $z$, i.e.,

$$
T_{z}^{c}(b M)=\left\{w=\left(w_{1}, \ldots, w_{n}\right) \in T_{z}(N): \sum_{j=1}^{n} \frac{\partial \rho}{\partial z_{j}}(z) w_{j}=0\right\} .
$$

Communicated by K. Saito. Received November 28, 2005. Revised October 16, 2006, January 18, 2007.

2000 Mathematics Subject Classification(s): Primary 32T15; Secondary 32T40, 46 E15.

Key words and phrases: Covering, holomorphic $L^{2}$ function, strongly pseudoconvex manifold.

Research supported in part by NSERC.

* Department of Mathematics and Statistics, University of Calgary, 2500 University Drive N.W., Calgary, Alberta T2N 1N4, Canada.

e-mail: albru@math.ucalgary.ca

(c) 2007 Research Institute for Mathematical Sciences, Kyoto University. All rights reserved. 
The Levi form of $\rho$ at $z \in b M$ is a hermitian form on $T_{z}^{c}(b M)$ defined in the local coordinates by the formula

$$
L_{z}(w, \bar{w})=\sum_{j, k=1}^{n} \frac{\partial^{2} \rho}{\partial z_{j} \partial \bar{z}_{k}}(z) w_{j} \bar{w}_{k} .
$$

The manifold $M$ is called pseudoconvex if $L_{z}(w, \bar{w}) \geq 0$ for all $z \in b M$ and $w \in T_{z}^{c}(b M)$. It is called strongly pseudoconvex if $L_{z}(w, \bar{w})>0$ for all $z \in b M$ and all $w \neq 0, w \in T_{z}^{c}(b M)$.

Equivalently, strongly pseudoconvex manifolds can be described as the ones which locally, in a neighbourhood of any boundary point, can be presented as strictly convex domains in $\mathbb{C}^{n}$. It is also known (see $[\mathrm{C}],[\mathrm{R}]$ ) that any strongly pseudoconvex manifold admits a proper holomorphic map with connected fibres onto a normal Stein space.

Without loss of generality we may and will assume that $\pi_{1}(M)=\pi_{1}(N)$ for $M$ as above. Let $r: N^{\prime} \rightarrow N$ be an unbranched covering of $N$. By $M^{\prime}:=$ $r^{-1}(M)$ we denote the corresponding covering of $M$. Also, by $b M^{\prime}:=r^{-1}(b M)$ and $\overline{M^{\prime}}:=M^{\prime} \cup b M^{\prime}$ we denote the boundary and the closure of $M^{\prime}$ in $N^{\prime}$.

Let $d V_{M^{\prime}}$ be the Riemannian volume form on $M^{\prime}$ obtained by a Riemannian metric pulled back from $N$. By $H^{2}\left(M^{\prime}\right)$ we denote the Hilbert space of holomorphic functions $g$ on $M^{\prime}$ with norm

$$
\left(\int_{z \in M^{\prime}}|g(z)|^{2} d V_{M^{\prime}}(z)\right)^{1 / 2} .
$$

Let $X$ be a subspace of the space $\mathcal{O}\left(M^{\prime}\right)$ of all holomorphic functions on $M^{\prime}$.

A point $z \in b M^{\prime}$ is called a peak point for $X$ if there exists a function $f \in X$ such that $f$ is unbounded on $M^{\prime}$ but bounded outside $U \cap M^{\prime}$ for any neighbourhood $U$ of $z$ in $N^{\prime}$.

A point $z \in b M^{\prime}$ is called a local peak point for $X$ if there exists a function $f \in X$ such that $f$ is unbounded in $U \cap M^{\prime}$ for any neighbourhood $U$ of $z$ in $N^{\prime}$ and there exists a neighbourhood $U$ of $z$ in $N^{\prime}$ such that for any neighbourhood $V$ of $z$ in $N^{\prime}$ the function $f$ is bounded on $U \backslash V$.

The Oka-Grauert theorem [G] implies that if $M$ is strongly pseudoconvex and $b M$ is not empty then every $z \in b M$ is a peak point for $H^{2}(M)$. In general it is not known whether a similar statement is true for boundary points of an infinite covering $M^{\prime}$ of $M$.

Assume that $M^{\prime}:=M_{G}$ is a regular covering of $M$ with a transformation group $G$. In $[\mathrm{GHS}]$ the von Neumann $G$-dimension $\operatorname{dim}_{G}$ was used to measure 
the space $H^{2}\left(M_{G}\right)$. In particular, one of the main results [GHS, Theorem 0.2] states:

Theorem. If $M$ is strongly pseudoconvex, then

(a) $\operatorname{dim}_{G} H^{2}\left(M_{G}\right)=\infty$ and

(b) each point in $b M_{G}$ is a local peak point for $H^{2}\left(M_{G}\right)$.

Also, in [GHS, p. 554] the following important question was asked: " $A$ natural question arises: is the compact group action really relevant for the existence of many holomorphic $L^{2}$-functions or is it just an artifact of the chosen methods which require a use of von Neumann algebras?" And further: "It is not clear how to formulate conditions assuring that $\operatorname{dim} L^{2} \mathcal{O}\left(M^{\prime}\right)=\infty^{1}$ without any group action."

In fact, in the above question the authors do not mean coverings of strongly pseudoconvex manifolds only but rather complex manifolds of bounded geometry satisfying certain geometrical conditions.

The main result of the present paper answers the above formulated question in the case of coverings of strongly pseudoconvex manifolds. In particular, we show that the regularity of $M^{\prime}$ is irrelevant for the existence of many holomorphic $L^{2}$ functions on $M^{\prime}$. Moreover, we also prove an extension of the above result of [GHS]. Our method of the proof is different from that used in [GHS] and is based on the $L^{2}$ cohomology techniques, as well as, on the geometric properties of $M$.

1.2. To formulate our result, let $C_{M} \subset M$ be the union of all compact complex subvarieties of $M$ of complex dimension $\geq 1$. It is known that if $M$ is strongly pseudoconvex, then $C_{M}$ is a compact complex subvariety of $M$. Let $z_{i}, 1 \leq$ $i \leq m$, be distinct points in $M \backslash C_{M}$. By $l_{2}\left(z_{i}^{\prime}\right)$ we denote the Hilbert space of $l_{2}$ functions on the fibre $z_{i}^{\prime}:=r^{-1}\left(z_{i}\right)$.

Theorem 1.1. If $M$ is strongly pseudoconvex, then

(a) For any $f_{i} \in l_{2}\left(z_{i}^{\prime}\right), 1 \leq i \leq m$, there exists $F \in H^{2}\left(M^{\prime}\right)$ such that $\left.F\right|_{z_{i}^{\prime}}=f_{i}, 1 \leq i \leq m$

(b) Each point in $b M^{\prime}$ is a peak point for $H^{2}\left(M^{\prime}\right)$.

Similar results are valid for certain weighted $L^{p}$ spaces of holomorphic functions on $M^{\prime}$. These and some other results will be published elsewhere. It

\footnotetext{
${ }^{1} \operatorname{dim} H^{2}\left(M^{\prime}\right)$ in our notation
} 
is worth noting that results much stronger than Theorem 1.1 can be obtained if $M$ is a strongly pseudoconvex Stein manifold, see [Br1], [Br2] for an exposition.

1.3. One of the purposes of [GHS] and of the present paper was to make a contribution toward settling the Shafarevich conjecture. Let us recall that the Shafarevich conjecture states that the universal covering $\widetilde{L}$ of a complex projective manifold $L$ is holomorphically convex meaning that for every infinite sequence without limit points in $\widetilde{L}$ there exists a holomorphic function on $\widetilde{L}$ that is unbounded on this sequence. According to a theorem of Grauert any complex projective manifold $L$ admits a holomorphic embedding into a strongly pseudoconvex manifold $M$ of complex dimension $\operatorname{dim}_{\mathbb{C}} M=\operatorname{dim}_{\mathbb{C}} L+1$ with the same fundamental group. Thus the basic idea is to try to develop the complex analysis on coverings $M^{\prime}$ of $M$ and then, taking restrictions of holomorphic functions on $M^{\prime}$ to the corresponding coverings $L^{\prime}\left(\subset M^{\prime}\right)$, to study holomorphic functions on $L^{\prime}$.

The results of this paper and [GHS] don't imply directly any new results in the area of the Shafarevich conjecture. However, one obtains a rich complex function theory on coverings of strongly pseudoconvex manifolds $M^{\prime}$ (as above). Thus there is the hope that together with some additional ideas and methods it could give some information about holomorphic functions on $L^{\prime}$. We are planning to return to this matter in a forthcoming paper.

Concerning the Shafarevich conjecture, for now the strongest results in this area are due to Eyssidieux [E] and, independently, to Campana [Ca] and the author [Br3]. The main result of the first of these papers states that the regular covering of a complex projective manifold $L$ corresponding to the intersection of the kernels of all linear reductive representations $\pi_{1}(L) \rightarrow G L_{n}(\mathbb{C})$, with a fixed $n$, is holomorphically convex. In the last papers it is proved that the regular covering of a complex projective manifold $L$ corresponding to the intersection of the kernels of all representations of $\pi_{1}(L)$ to complex solvable Lie groups is holomorphically convex. For other fundamental results in the area of the Shafarevich conjecture we refer the reader to the Featured Review by Katzarkov of the paper $[\mathrm{E}]$.

\section{§2. Auxiliary Results}

2.1. Let $X$ be a complete Kähler manifold of dimension $n$ with a Kähler form $\omega$ and $E$ be a hermitian holomorphic vector bundle on $X$ with curvature $\Theta$. Let $L_{2}^{p, q}(X, E)$ be the space of $L^{2} E$-valued $(p, q)$-forms on $X$ with the $L^{2}$ norm, and let $W_{2}^{p, q}(X, E)$ be the subspace of forms such that $\bar{\partial} \eta$ is $L^{2}$. (The forms 
$\eta$ may be taken to be either smooth or just measurable, in which case $\bar{\partial} \eta$ is understood in the distributional sense.) The cohomology of the resulting $L^{2}$ Dolbeault complex $\left(W_{2}^{, \cdot}, \bar{\partial}\right)$ is the $L^{2}$ cohomology

$$
H_{(2)}^{p, q}(X, E)=Z_{2}^{p, q}(X, E) / B_{2}^{p, q}(X, E),
$$

where $Z_{2}^{p, q}(X, E)$ and $B_{2}^{p, q}(X, E)$ are the spaces of $\bar{\partial}$-closed and $\bar{\partial}$-exact forms in $L_{2}^{p, q}(X, E)$, respectively.

If $\Theta \geq \epsilon \omega$ for some $\epsilon>0$ in the sense of Nakano, then the $L^{2}$ KodairaNakano vanishing theorem, see $[\mathrm{D}],[\mathrm{O}]$, states that

$$
H_{(2)}^{n, r}(X, E)=0 \quad \text { for } \quad r>0
$$

2.2. Let $M \subset \subset N$ be a strongly pseudoconvex manifold. Without loss of generality we will assume that $\pi_{1}(M)=\pi_{1}(N)$ and $N$ is strongly pseudoconvex, as well. Then there exist a normal Stein space $X_{N}$, a proper holomorphic surjective map $p: N \rightarrow X_{N}$ with connected fibres and points $x_{1}, \ldots, x_{l} \in X_{N}$ such that

$$
p: N \backslash \bigcup_{1 \leq i \leq l} p^{-1}\left(x_{i}\right) \rightarrow X_{N} \backslash \bigcup_{1 \leq i \leq l}\left\{x_{i}\right\}
$$

is biholomorphic, see [C], [R]. By definition, the domain $X_{M}:=p(M) \subset X_{N}$ is strongly pseudoconvex, and so it is Stein. Without loss of generality we may assume that $x_{1}, \ldots, x_{l} \in X_{M}$. Thus $\cup_{1 \leq i \leq l} p^{-1}\left(x_{i}\right)=C_{M}$.

Let $L \subset \subset N$ be a strongly pseudoconvex neighbourhood of $\bar{M}$. Then $X_{L}:=p(L)$ is a strongly pseudoconvex neighbourhood of $\bar{X}_{M}$ in $X_{N}$. We introduce a complete Kähler metric on the complex manifold $L \backslash C_{M}$ as follows.

According to $[\mathrm{N}]$ there is a proper one-to-one holomorphic map $i: X_{L} \hookrightarrow$ $\mathbb{C}^{2 n+1}, n=\operatorname{dim}_{\mathbb{C}} X_{L}$, which is an embedding at regular points of $X_{L}$. Thus $i\left(X_{L}\right) \subset \mathbb{C}^{2 n+1}$ is a closed complex subvariety. By $\omega_{e}$ we denote the $(1,1)$-form on $L$ obtained as the pullback by $i \circ p$ of the Euclidean Kähler form on $\mathbb{C}^{2 n+1}$. Clearly, $\omega_{e}$ is $d$-closed and positive outside $C_{M}$.

Similarly we can embed $X_{N}$ into $\mathbb{C}^{2 n+1}$ as a closed complex subvariety. Let $j: X_{N} \hookrightarrow \mathbb{C}^{2 n+1}$ be an embedding such that $j\left(X_{L}\right)$ belongs to the open Euclidean ball $B$ of radius $1 / 4$ centered at $0 \in \mathbb{C}^{2 n+1}$. Set $z_{i}:=j\left(x_{i}\right), 1 \leq i \leq l$. By $\omega_{i}$ we denote the restriction to $L \backslash C_{M}$ of the pullback with respect to $j \circ p$ of the form $-\sqrt{-1} \cdot \partial \bar{\partial} \log \left(\log \left\|z-z_{i}\right\|^{2}\right)^{2}$ on $\mathbb{C}^{2 n+1} \backslash\left\{z_{i}\right\}$. (Here $\|\cdot\|$ stands for the Euclidean norm on $\mathbb{C}^{2 n+1}$.) Since $j\left(X_{L}\right) \subset B$, the form $\omega_{i}$ is Kähler. Its positivity follows from the fact that the function $-\log \left(\log \|z\|^{2}\right)^{2}$ is strictly 
plurisubharmonic for $\|z\|<1$. Also, $\omega_{i}$ is extended to a smooth form on $L \backslash p^{-1}\left(x_{i}\right)$. Now, let us introduce a Kähler form $\omega_{L}$ on $L \backslash C_{M}$ by the formula

$$
\omega_{L}:=\omega_{e}+\sum_{1 \leq i \leq l} \omega_{i}
$$

Proposition 2.1. The path metric d on $L \backslash C_{M}$ induced by $\omega_{L}$ is complete.

Proof. Assume, on the contrary, that there is a sequence $\left\{w_{j}\right\}$ convergent either to $C_{M}$ or to the boundary $b L$ of $L$ such that the sequence $\left\{d\left(o, w_{j}\right)\right\}$ is bounded (for a fixed point $o \in L \backslash C_{M}$ ). Then, since $\omega_{L} \geq \omega_{e}$, the sequence $\left\{i\left(p\left(w_{j}\right)\right)\right\} \subset \mathbb{C}^{2 n+1}$ is bounded. This implies that $\left\{w_{j}\right\}$ converges to $C_{M}$. But since $\omega_{L} \geq \sum \omega_{i}$, the latter is impossible, see, e.g., [GM] for similar arguments.

In the same way one obtains complete Kähler metrics on unbranched coverings of $L \backslash C_{M}$ induced by pullbacks to these coverings of the Kähler form $\omega_{L}$ on $L \backslash C_{M}$.

2.3. We retain the notation of the previous section. Also, for an $n$-dimensional complex manifold $X$ by $T_{X}$ and $T_{X}^{*}$ we denote complex tangent and cotangent bundles on $X$ and by $K_{X}=\wedge^{n} T_{X}^{*}$ the canonical line bundle on $X$.

Let $r: N^{\prime} \rightarrow N$ be an unbranched covering. Consider the corresponding covering $\left(L \backslash C_{M}\right)^{\prime}:=r^{-1}\left(L \backslash C_{M}\right)$ of $L \backslash C_{M}$. We equip $\left(L \backslash C_{M}\right)^{\prime}$ with the complete Kähler metric induced by the form $\omega_{L}^{\prime}:=r^{*} \omega_{L}$.

Next we consider the function $f:=\sum_{0 \leq i \leq l} f_{i}$ on $\left(L \backslash C_{M}\right)^{\prime}$ such that $f_{0}$ is the pullback by $i \circ p \circ r$ of the function $\|z\|^{2}$ on $\mathbb{C}^{2 n+1}$ and $f_{i}$ is the pullback by $j \circ p \circ r$ of the function $-\log \left(\log \left\|z-z_{i}\right\|^{2}\right)^{2}$ on $\mathbb{C}^{2 n+1} \backslash\left\{z_{i}\right\}, 1 \leq i \leq l$. Clearly we have

$$
\omega_{L}^{\prime}:=\sqrt{-1} \cdot \partial \bar{\partial} f
$$

Let $E:=\left(L \backslash C_{M}\right)^{\prime} \times \mathbb{C}$ be the trivial holomorphic line bundle on $\left(L \backslash C_{M}\right)^{\prime}$. Let $g$ be the pullback to $\left(L \backslash C_{M}\right)^{\prime}$ of a smooth plurisubharmonic function on $L$. We equip $E$ with the hermitian metric $e^{-f-g}$ (i.e., for $z \times v \in E$ the square of its norm in this metric equals $e^{-f(z)-g(z)}|v|^{2}$ where $|v|$ is the modulus of $v \in \mathbb{C})$. Then the curvature $\Theta_{E}$ of $E$ satisfies

$$
\Theta_{E}:=-\sqrt{-1} \cdot \partial \bar{\partial} \log \left(e^{-f-g}\right)=\omega_{L}^{\prime}+\sqrt{-1} \cdot \partial \bar{\partial} g \geq \omega_{L}^{\prime} .
$$


Thus we can apply the $L^{2}$ Kodaira-Nakano vanishing theorem of Section 2.1 to get

$$
H_{(2)}^{n, r}\left(\left(L \backslash C_{M}\right)^{\prime}, E\right)=0 \quad \text { for } \quad r>0 .
$$

Let $K_{\left(L \backslash C_{M}\right)^{\prime}}$ be the canonical holomorphic line bundle on $\left(L \backslash C_{M}\right)^{\prime}$ equipped with the hermitian metric induced by $\omega_{L}^{\prime}$. Consider the hermitian line bundle $V_{g}:=E \otimes K_{\left(L \backslash C_{M}\right)^{\prime}}$ equipped with the tensor product of the corresponding hermitian metrics. Then from (2.5) we have

$$
H_{(2)}^{0, r}\left(\left(L \backslash C_{M}\right)^{\prime}, V_{g}\right) \cong H_{(2)}^{n, r}\left(\left(L \backslash C_{M}\right)^{\prime}, E\right)=0 \quad \text { for } \quad r>0 .
$$

2.4. Let $U \subset L$ be a relatively compact neighbourhood of $C_{M}$. Consider a finite open cover $\left(U_{i}\right)_{1 \leq i \leq k}$ of $\overline{L \backslash U}$ by simply connected coordinate charts $U_{i} \subset \subset N \backslash C_{M}$. We introduce complex coordinates on $U_{i}^{\prime}:=r^{-1}\left(U_{i}\right) \subset N^{\prime}$ by the pullback of the coordinates on $U_{i}$. In these coordinates $U_{i}^{\prime}$ is naturally identified with $U_{i} \times S$ where $S$ is the fibre of $r: N^{\prime} \rightarrow N$.

Let $\eta$ be a smooth $(p, q)$-form on $\left(L \backslash C_{M}\right)^{\prime}$ equals 0 on $r^{-1}(U)$. Then in the above holomorphic coordinates $(z, s), z=\left(z_{1}, \ldots, z_{n}\right) \in U_{i} \cap L, s \in S$, on $U_{i}^{\prime} \cap L^{\prime}, L^{\prime}:=r^{-1}(L)$, the form $\eta$ is presented as

$$
\eta(z, s)=\sum_{i_{1}, \ldots, i_{p}, j_{1}, \ldots, j_{q}} \eta_{i ; i_{1}, \ldots, i_{p}, j_{1}, \ldots, j_{q}}(z, s) d z_{i_{1}} \wedge \cdots \wedge d z_{i_{p}} \wedge d \bar{z}_{j_{1}} \wedge \cdots \wedge d \bar{z}_{j_{q}}
$$

where $\eta_{i ; i_{1}, \ldots, i_{p}, j_{1}, \ldots, j_{q}}$ are smooth functions on $\left(U_{i} \cap L\right) \times S$.

We say that $\eta$ belongs to the space $\mathcal{E}_{U ; 2}^{p, q}\left(\left(L \backslash C_{M}\right)^{\prime}\right)$ if in (2.7) we have

$$
\sup _{z \in U_{i} \cap L, i, i_{1}, \ldots, i_{p}, j_{1}, \ldots, j_{q}}\left\{\sum_{s \in S}\left|\eta_{i ; i_{1}, \ldots, i_{p}, j_{1}, \ldots, j_{q}}(z, s)\right|^{2}\right\}<\infty .
$$

Let $e$ be a holomorphic section of $\left.K\right|_{L \backslash C_{M}}$. Then $\eta \cdot r^{*} e$ can be viewed as a $(p, q)$-form with values in $V_{g}$. (Here $r^{*} e$ is the pullback of $e$ to $\left(L \backslash C_{M}\right)^{\prime}$, i.e., $r^{*} e \in \mathcal{O}\left(\left(L \backslash C_{M}\right)^{\prime},\left.K\right|_{\left.\left.\left(L \backslash C_{M}\right)^{\prime}\right) .\right)}\right.$

Proposition 2.2. For every $\eta \in \mathcal{E}_{U ; 2}^{p, q}\left(\left(L \backslash C_{M}\right)^{\prime}\right)$ and $e \in \mathcal{O}\left(L \backslash C_{M}\right.$, $\left.\left.K\right|_{L \backslash C_{M}}\right)$ there is a plurisubharmonic function $g$ in the definition of the metric on $V_{g}$ such that $\eta \cdot r^{*} e \in L_{2}^{p, q}\left(\left(L \backslash C_{M}\right)^{\prime}, V_{g}\right)$.

Proof. In this proof by $\|\cdot\|$ we denote the hermitian metric on the space of $V_{g}$-valued $(p, q)$-forms induced by the hermitian metrics on $V_{g}$ and $T_{\left(L \backslash C_{M}\right)^{\prime}}$. Set

$$
h_{i ; i_{1}, \ldots, i_{p}, j_{1}, \ldots, j_{q}}(z):=\left\|r^{*} e(z, s) \cdot d z_{i_{1}} \wedge \cdots \wedge d z_{i_{p}} \wedge d \bar{z}_{j_{1}} \wedge \cdots \wedge d \bar{z}_{j_{q}}\right\|^{2} .
$$


Then $h_{i ; i_{1}, \ldots, i_{p}, j_{1}, \ldots, j_{q}}$ is a nonnegative continuous function on $U_{i} \cap L$. Let $\hat{g}$ be such that $r^{*} \hat{g}=g$. By the definition of metrics on $V_{g}$ and $T_{\left(L \backslash C_{M}\right)^{\prime}}$

$$
h_{i ; i_{1}, \ldots, i_{p}, j_{1}, \ldots, j_{q}}(z):=\hat{h}_{i ; i_{1}, \ldots, i_{p}, j_{1}, \ldots, j_{q}}(z) \cdot e^{-\hat{g}(z)},
$$

where $\hat{h}_{i ; i_{1}, \ldots, i_{p}, j_{1}, \ldots, j_{q}}$ is a nonnegative continuous function on $U_{i} \cap L$ independent of $\hat{g}$.

Now for some $A \in \mathbb{N}$ we have

$$
\begin{aligned}
& \left\|\eta(z, s) \cdot r^{*} e(z, s)\right\|^{2} \\
\leq & A \times \sum_{i_{1}, \ldots, i_{p}, j_{1}, \ldots, j_{q}}\left|\eta_{i ; i_{1}, \ldots, i_{p}, j_{1}, \ldots, j_{q}}(z, s)\right|^{2} \cdot h_{i ; i_{1}, \ldots, i_{p}, j_{1}, \ldots, j_{q}}(z) .
\end{aligned}
$$

According to the definition of $L_{2}^{p, q}\left(\left(L \backslash C_{M}\right)^{\prime}, V_{g}\right)$ we have to show that

$$
\left|\eta \cdot r^{*} e\right|^{2}:=\int_{\left(L \backslash C_{M}\right)^{\prime}}\left\|\eta \cdot r^{*} e\right\|^{2} \cdot\left(\omega_{L}^{\prime}\right)^{n}<\infty .
$$

Since $\omega_{L}^{\prime}=r^{*} \omega_{L}$, from (2.9) and (2.10) we get

$$
\begin{aligned}
& \left|\eta \cdot r^{*} e\right|^{2} \leq \\
& A \times \sum_{i=1}^{k} \int_{U_{i} \cap L}\left(\sum_{i_{1}, \ldots, i_{p}, j_{1}, \ldots, j_{q}, s \in S}\left|\eta_{i ; i_{1}, \ldots, i_{p}, j_{1}, \ldots, j_{q}}(\cdot, s)\right|^{2}\right) \hat{h}_{i ; i_{1}, \ldots, i_{p}, j_{1}, \ldots, j_{q}} e^{-\hat{g}} \omega_{L}^{n} .
\end{aligned}
$$

Also, by the hypothesis of the proposition, see (2.8),

$$
\sup _{z \in U_{i} \cap L}\left\{\sum_{i_{1}, \ldots, i_{p}, j_{1}, \ldots, j_{q}, s \in S}\left|\eta_{i ; i_{1}, \ldots, i_{p}, j_{1}, \ldots, j_{q}}(z, s)\right|^{2}\right\}<\infty \quad \text { for } \quad 1 \leq i \leq k .
$$

Thus in order to prove the proposition it suffices to check that there is $\hat{g}$ in the definition of the metric on $V_{g}$ such that for every $i$

$$
\int_{U_{i} \cap L} \hat{h}_{i ; i_{1}, \ldots, i_{p}, j_{1}, \ldots, j_{q}} e^{-\hat{g}} \omega_{L}^{n}<\infty .
$$

The required result now follows from

Lemma 2.3. Let $h$ be a nonnegative piecewise continuous function on $L$ equals 0 in some neighbourhood of $C_{M}$ and bounded on every compact subset of $L \backslash C_{M}$. Then there exists a smooth plurisubharmonic function $\hat{g}$ on $L$ such that

$$
\int_{L} h \cdot e^{-\hat{g}} \omega_{L}^{n}<\infty
$$


Proof. Without loss of generality we identify $L \backslash C_{M}$ with $X_{L} \backslash$ $\cup_{1 \leq j \leq l}\left\{x_{j}\right\}$. Also, we identify $X_{L}$ with a closed subvariety of $\mathbb{C}^{2 n+1}$ as in Section 2.2. Let $U$ be a neighbourhood of $\cup_{1 \leq j \leq l}\left\{x_{j}\right\}$ such that $\left.h\right|_{U} \equiv 0$. By $\Delta_{r} \subset \mathbb{C}^{2 n+1}$ we denote the open polydisk of radius $r$ centered at $0 \in \mathbb{C}^{2 n+1}$. Assume without loss of generality that $0 \in X_{L} \backslash U$. Consider the monotonically increasing function

$$
v(r):=\int_{\Delta_{r} \cap\left(X_{L} \backslash U\right)} h \cdot \omega_{L}^{n}, \quad r \geq 0 .
$$

By $v_{1}$ we denote a smooth monotonically increasing function satisfying $v \leq v_{1}$ ( $\operatorname{such} v_{1}$ can be easily constructed by $v$ ). Let us determine

$$
v_{2}(r):=\int_{0}^{r+1} 2 v_{1}(2 t) d t, \quad r \geq 0 .
$$

By the definition $v_{2}$ is smooth, convex and monotonically increasing. Moreover,

$$
v_{2}(r) \geq \int_{\frac{r+1}{2}}^{r+1} 2 v_{1}(2 t) d t \geq(r+1) v(r+1) .
$$

Next we define a smooth plurisubharmonic function $v_{3}$ on $\mathbb{C}^{2 n+1}$ by the formula

$$
v_{3}\left(z_{1}, \ldots, z_{2 n+1}\right):=\sum_{j=1}^{2 n+1} v_{2}\left(\left|z_{j}\right|\right) .
$$

Then the pullback of $v_{3}$ to $L$ is a smooth plurisubharmonic function on $L$. This is the required function $\hat{g}$. Indeed, under the identification described at the beginning of the proof we have

$$
\begin{gathered}
\int_{L} h \cdot e^{-\hat{g}} \omega_{L}^{n}=\sum_{k=1}^{\infty} \int_{\left(\Delta_{k} \backslash \Delta_{k-1}\right) \cap\left(X_{L} \backslash U\right)} h \cdot e^{-\hat{g}} \omega_{L}^{n} \leq \\
\sum_{k=1}^{\infty} v(k) e^{-v_{2}(k-1)} \leq \sum_{k=1}^{\infty} v(k) e^{-k v(k)}<\infty .
\end{gathered}
$$

To complete the proof of the proposition it remains to put in the above lemma

$$
h:=\sum_{i, i_{1}, \ldots, i_{p}, j_{1}, \ldots, j_{q}} \rho_{i} \cdot \hat{h}_{i ; i_{1}, \ldots, i_{p}, j_{1}, \ldots, j_{q}}
$$

where $\rho_{i}$ is the characteristic function of $U_{i} \cap L$. 
2.5. Let $O \subset \subset L$ be a neighbourhood of $C_{M}$. We set $O^{\prime}:=r^{-1}(O), C_{M}^{\prime}:=$ $r^{-1}\left(C_{M}\right)$. Assume that the manifold $N$, see Section 1.1, is equipped with a hermitian metric $\rho$. We equip the bundle $K_{L}$ with the hermitian metric induced by $\rho$. Also, we equip $K_{L^{\prime}}:=r^{*} K_{L}$ with the hermitian metric $\rho^{\prime}:=r^{*} \rho$.

Proposition 2.4. Any $h \in L_{2}\left(\left(L \backslash C_{M}\right)^{\prime}, V_{g}\right)$ holomorphic on $O^{\prime} \backslash C_{M}^{\prime}$ admits an extension to a section $\hat{h}$ of $K_{L^{\prime}}$ such that $\left.\hat{h}\right|_{M^{\prime}} \in L_{2}\left(M^{\prime}, K_{L^{\prime}}\right)$.

Proof. Consider a coordinate neighbourhood $U \subset \subset O$ of a point $q \in C_{M}$ with coordinates $z=\left(z_{1}, \ldots, z_{n}\right)$. Taking the pullback of these coordinates to $r^{-1}(U)$ we identify $r^{-1}(U)$ with $U \times S$ where $S$ is the fibre of $r$. Then

$$
h(z, s)=h_{U}(z, s) d z_{1} \wedge \cdots \wedge d z_{n}, \quad z \in U \backslash C_{M}, s \in S .
$$

By the definition of the metric $\|\cdot\|$ on $V_{g}$ we have

$$
\begin{gathered}
\|h(z, s)\|^{2} \omega_{L}^{n}(z)=\left|h_{U}(z, s)\right|^{2}\left\|d z_{1} \wedge \cdots \wedge d z_{n}\right\|^{2} \omega_{L}^{n}(z)= \\
\left|h_{U}(z, s)\right|^{2} e^{-\hat{g}(z)}(\sqrt{-1})^{n} \wedge_{i=1}^{n} d z_{i} \wedge d \bar{z}_{i} .
\end{gathered}
$$

Now, the hypotheses of the proposition imply that

$$
\int_{z \in U \backslash C_{M}}\left(\sum_{s \in S}\left|h_{U}(z, s)\right|^{2}\right) e^{-\hat{g}(z)}(\sqrt{-1})^{n} \wedge_{i=1}^{n} d z_{i} \wedge d \bar{z}_{i}<\infty .
$$

Let $\omega^{n}$ be the volume form induced by the hermitian metric $\rho$ on $N$ with the associated $(1,1)$-form $\omega$. Since by our construction $\hat{g}$ is smooth on $L$, we have on $U$ :

$$
e^{-\hat{g}(z)}(\sqrt{-1})^{n} \wedge_{i=1}^{n} d z_{i} \wedge d \bar{z}_{i} \geq c \omega^{n}
$$

for some $c>0$. From here and (2.13) we get

$$
\int_{z \in U \backslash C_{M}}\left(\sum_{s \in S}\left|h_{U}(z, s)\right|^{2}\right) \omega^{n}(z)<\infty .
$$

In particular, this implies that every $h_{U}(\cdot, s), s \in S$, belongs to the $L^{2}$ space on $U \backslash C_{M}$ defined by integration with respect to the volume form $(\sqrt{-1})^{n} \wedge_{i=1}^{n}$ $d z_{i} \wedge d \bar{z}_{i}$. Also, every $h_{U}(\cdot, s)$ is holomorphic on $U \backslash C_{M}$. Using these facts and the Cauchy integral formulas for coefficients of the Laurent expansion of $h_{U}(\cdot, s)$, one obtains easily that every $h_{U}(\cdot, s)$ can be extended holomorphically to $U$. In turn, this gives an extension $\hat{h}$ of $h$ to $r^{-1}(U)$. Now from (2.14) we obtain that $\hat{h} \in L_{2}\left(U, K_{L^{\prime}}\right)$. 
Next assume that $\widetilde{U} \subset \subset \backslash \backslash C_{M}$ is a simply connected coordinate neighbourhood of a point $q \in \bar{M} \backslash C_{M}$. Identifying $r^{-1}(\widetilde{U})$ with $\widetilde{U} \times S$ we have anew inequality of type (2.13) for $\left.h\right|_{r^{-1}(\widetilde{U})}$. Since $\hat{g}$ is smooth on $L \backslash C_{M}$, repeating literally the previous arguments we get that $h \in L_{2}\left(\widetilde{U}, K_{L^{\prime}}\right)$. Taking a finite open cover of $\bar{M}$ by the above neighbourhoods $U$ and $\widetilde{U}$ and considering the extension of $h$ to $M^{\prime}$ defined by the above extended forms $\hat{h}$ on $r^{-1}(U)$ we get the required result.

\section{$\S 3 . \quad$ Proofs}

3.1. Proof of Theorem 1.1 (a). First, we prove Theorem 1.1 (a) for $m=1$ :

Theorem 3.1. Let $z \in M \backslash C_{M}$ and $z^{\prime}:=r^{-1}(z) \in M^{\prime}$. Then for any $f \in l_{2}\left(z^{\prime}\right)$ there exists $F \in H^{2}\left(M^{\prime}\right)$ such that $\left.F\right|_{z^{\prime}}=f$.

Proof. In the proof we retain the notation of Section 2.

Let $p: N \rightarrow X_{N}$ be the proper holomorphic map onto the normal Stein space $X_{N}$ from Section 2.2 such that $p: N \backslash C_{M} \rightarrow X_{N} \backslash \cup_{1 \leq i \leq l}\left\{x_{i}\right\}$ is biholomorphic. Since $X_{N}$ is Stein, there is a holomorphic function $h$ on $X_{N}$ whose set of zeros $\mathcal{Z}_{h}$ contains $p(z)$ and does not intersect $\cup_{1 \leq j \leq l}\left\{x_{j}\right\}$. Let $O \subset \subset X_{N}$ be a Stein neighbourhood of the compact set $\mathcal{Z}_{h} \cap \bar{X}_{L}, X_{L}:=p(L)$, such that $\bar{O} \cap \cup_{1 \leq j \leq l}\left\{x_{j}\right\}=\emptyset$ and $\bar{O}$ is holomorphically convex in $X_{N}$. We set $O^{\prime}:=(p \circ r)^{-1}(O) \subset N^{\prime}$. Then according to [Br1, Theorem 1.10] there is a holomorphic function $h_{1}$ on $O^{\prime}$ satisfying

$$
\left.h_{1}\right|_{z^{\prime}}=f \quad \text { and } \sup _{y \in p^{-1}(O)}\left\{\sum_{x \in r^{-1}(y)}\left|h_{1}(x)\right|^{2}\right\}<\infty .
$$

Let $\rho$ be a $C^{\infty}$ function on $X_{N} \backslash \cup_{1 \leq i \leq l}\left\{x_{i}\right\}$ equals 1 in some neighbourhood of $\mathcal{Z}_{h} \cap O$ in $O$ and 0 outside $O$. By $\rho^{\prime}:=(p \circ r)^{*} \rho$ we denote its pullback to $N^{\prime}$. Then $h_{2}:=\left.\left(\rho^{\prime} \cdot h_{1}\right)\right|_{\left(L \backslash C_{M}\right)^{\prime}}$ is a $C^{\infty}$ function on $\left(L \backslash C_{M}\right)^{\prime}$. Let $h^{\prime}:=\left.(p \circ r)^{*} h\right|_{L^{\prime}}$ be the restriction to $L^{\prime}$ of the pullback of $h$. Consider the $C^{\infty}(0,1)$-from $\eta:=\bar{\partial} h_{2} / h^{\prime}$. It follows easily from (3.1) and (2.8) that $\eta \in \mathcal{E}_{U ; 2}^{0,1}\left(\left(L \backslash C_{M}\right)^{\prime}\right)$ for some $U \subset L \backslash p^{-1}(O)$.

Next, since $X_{N}$ can be embedded to $\mathbb{C}^{2 n+1}$, and since $z$ is a regular point of $X_{N}$, there is a section $e \in \mathcal{O}\left(N, K_{N}\right)$ such that $e(z) \neq 0$. Let $V \subset L$ be the set of zeros of $e$. Then $V$ is contained in the preimage $p^{-1}\left(V^{\prime}\right)$ of a complex analytic subspace $V^{\prime} \subset X_{N}$. Since $X_{N}$ is Stein, the latter implies that there is a bounded holomorphic function $f_{e}$ on $L$ such that $f_{e} \cdot e^{-1}$ is a bounded section from $\mathcal{O}\left(L, K_{L}^{*}\right)$ and $f_{e}(z)=1$ (recall that $\left.L \subset \subset N\right)$. 
Further, by Proposition 2.2 there is a function $g$ in the definition of the metric on the vector bundle $V_{g}$ from Section 2.3 such that $\widetilde{\eta}:=\eta \cdot r^{*} e \in L_{2}^{0,1}((L \backslash$ $\left.\left.C_{M}\right)^{\prime}, V_{g}\right)$. Since $\bar{\partial} \widetilde{\eta}=0$, by $(2.6)$ there is a section $h_{3} \in L_{2}\left(\left(L \backslash C_{M}\right)^{\prime}, V_{g}\right)$ such that $\bar{\partial} h_{3}=\widetilde{\eta}$. Choose $g$ in the definition of the metric on $V_{g}$ so that also $h_{2} \cdot r^{*} e \in L_{2}\left(\left(L \backslash C_{M}\right)^{\prime}, V_{g}\right)$. Thus $h_{4}:=h_{2} \cdot r^{*} e-h^{\prime} h_{3} \in L_{2}((L \backslash$ $\left.\left.C_{M}\right)^{\prime}, V_{g}\right)$, is holomorphic on $\left(L \backslash C_{M}\right)^{\prime}$. By Proposition $2.4 h_{4}$ is extended to a holomorphic section $h_{4}^{\prime}$ of $K_{L^{\prime}}$ such that $\left.h_{4}^{\prime}\right|_{M^{\prime}} \in L_{2}\left(M^{\prime}, K_{L^{\prime}}\right)$. Moreover, by our construction $\left.h_{4}^{\prime}\right|_{z^{\prime}}=\left.f \cdot r^{*} e\right|_{z^{\prime}}$. Finally, we set

$$
F:=\left.\left.h_{4}^{\prime}\right|_{M^{\prime}} \cdot\left(r^{*} f_{e} \cdot r^{*} e^{-1}\right)\right|_{M^{\prime}} .
$$

Then $F \in H^{2}\left(M^{\prime}\right)$ and $\left.F\right|_{z^{\prime}}=f$.

Now, let us prove Theorem 1.1 (a).

By Theorem 3.1 there are functions $F_{i} \in H^{2}\left(M^{\prime}\right)$ such that $\left.F_{i}\right|_{z_{i}^{\prime}}=f_{i}$, $1 \leq i \leq m$. Let $t_{i}$ be a holomorphic function on $N$ such that $t_{i}\left(z_{i}\right)=1$ and $t_{i}\left(z_{j}\right)=0$ for $j \neq i$. Then the function $F:=\sum_{1 \leq i \leq m} r^{*} t_{i} \cdot F_{i}$ satisfies the required condition.

3.2. Proof of Theorem 1.1 (b). Let $x \in b M$ be a boundary point of a strongly pseudoconvex manifold $M \subset \subset N$. We will prove that each point $y \in r^{-1}(x)$ is a peak point for $H^{2}\left(M^{\prime}\right)$.

Choose a coordinate neighbourhood $U_{1} \subset \subset N$ of $x$ with complex coordinates $w=\left(w_{1}, \ldots, w_{n}\right)$ centered at $x$ such that the complex tangent space $T_{x}^{c}(b M)$ is given by the equation $w_{1}=0$. Consider the Taylor expansion of the defining function $\rho$ for $M$, see (1.1), (1.2), at $x$ :

$$
\rho(w)=\rho(x)+2 \operatorname{Re} f(x, w)+L_{x}(w-x, \bar{w}-\bar{x})+O\left(\|w-x\|^{3}\right),
$$

where $L_{x}$ is the Levi form at $x$ and $f(x, w)$ is a complex quadratic polynomial with respect to $w$ :

$$
f(x, w)=\frac{\partial \rho}{\partial w_{1}}(x) w_{1}+\frac{1}{2} \sum_{1 \leq \mu, \nu \leq n} \frac{\partial^{2} \rho}{\partial w_{\mu} \partial w_{\nu}}(x) w_{\mu} w_{\nu} .
$$

(Here $\frac{\partial \rho}{\partial w_{1}}(x) \neq 0$ and $\frac{\partial \rho}{\partial w_{i}}(x)=0$ for $2 \leq i \leq n$ by the choice of the coordinates on $\left.U_{1}\right)$. Next, in a sufficiently small simply connected neigbourhood $U \subset \subset U_{1}$ of $x$ we introduce new coordinates $z=\left(z_{1}, \ldots, z_{n}\right)$ by the formulas

$$
z_{1}=f(x, w) \quad \text { and } \quad z_{i}=w_{i} \text { for } \quad 2 \leq i \leq n .
$$

Since $b M$ is strongly pseudoconvex at $x$, diminishing if necessary $U$ we have that in $U$ the intersection of the hyperplane $z_{1}=0$ with $\bar{M}$ consists of one 
point $x$ and $\operatorname{Re} z_{1}<0$ in $U$. Let $H:=\left\{z \in U: \operatorname{Re} z_{1}<0\right\}$. Then we can choose a branch of $\log z_{1}$ so that $h_{x}(z)=\log z_{1}$ is a holomorphic function in $H$. Clearly, $h_{x} \in L^{2}(H)$ for $L^{2}$ defined by a Riemannian volume form pulled back from $N$, and $h_{x}$ has a peak point at $x$.

Let $H^{\prime}:=r^{-1}(H) \subset \subset N^{\prime}$. Take a point $y \in r^{-1}(x) \subset b M^{\prime}$. Let $H_{y}$ be the connected component of $H^{\prime}$ containing $y$. We set $h_{y}:=\left.r^{*} h_{x}\right|_{H_{y}}$. Next, consider a smooth function $\rho$ on $N$ equals 1 in a neigbourhood $O \subset \subset U$ of $x$ with support $S \subset \subset U$. Let $O_{y} \subset \subset U_{y}$ be connected components of $O^{\prime}=r^{-1}(O)$ and $U^{\prime}=r^{-1}(U)$ containing $y$. By $\rho_{y}$ we denote the pullback of $\left.\rho\right|_{U}$ to $U_{y}$ and by $S^{\prime} \subset \subset U_{y}^{\prime}$ support of $\rho_{y}$. Then $\bar{\partial}\left(h_{y} \rho_{y}\right)$ can be extended by 0 to a $C^{\infty}(0,1)$-form $\eta$ on $\left(N^{\prime} \backslash S^{\prime}\right) \cup H_{y}^{\prime} \cup O_{y}^{\prime}$. Note that $(N \backslash S) \cup H \cup O$ is a neighbourhood of $\bar{M}$. Hence, since $M \subset \subset N$ is strongly pseudoconvex, there is a strongly pseudoconvex manifold $L \subset \subset(N \backslash S) \cup H \cup O$ such that $\bar{M} \subset L$. Observe also that $r^{-1}((N \backslash S) \cup H \cup O) \subset\left(N^{\prime} \backslash S^{\prime}\right) \cup H_{y}^{\prime} \cup O_{y}^{\prime}$. Thus the form $\eta$ is well-defined on $L^{\prime}:=r^{-1}(L)$. Moreover, by our definitions $\eta \in \mathcal{E}_{W ; 2}^{0,1}\left(\left(L \backslash C_{M}\right)^{\prime}\right)$ for some neighbourhood $W$ of $C_{M}$, see Section 2.4.

As in the proof of part (a) we will choose a section $e \in \mathcal{O}\left(N, K_{N}\right)$ such that $e(x) \neq 0$ and a function $f_{e} \in \mathcal{O}(L)$ such that $f_{e}(x)=1$ and $f_{e} \cdot e^{-1}$ is a bounded section from $\mathcal{O}\left(L, K_{L}^{*}\right)$.

Now, by Proposition 2.2 there is a function $g$ in the definition of the metric on the vector bundle $V_{g}$ from Section 2.3 such that $\widetilde{\eta}:=\eta \cdot r^{*} e \in L_{2}^{0,1}((L \backslash$ $\left.\left.C_{M}\right)^{\prime}, V_{g}\right)$. Since $\bar{\partial} \widetilde{\eta}=0$, by $(2.6)$ there is a section $h_{1} \in L_{2}\left(\left(L \backslash C_{M}\right)^{\prime}, V_{g}\right)$ such that $\bar{\partial} h_{1}=\widetilde{\eta}$. According to Proposition $\left.2.4 h_{1}\right|_{\left(M \backslash C_{M}\right)^{\prime}}$ is extended to a section $h_{2}$ of $K_{L^{\prime}}$ such that $h_{2} \in L_{2}\left(M^{\prime}, K_{L^{\prime}}\right)$. From here, using the fact that $\left.\left(h_{y} \rho_{y}\right)\right|_{M^{\prime} \cap H_{y}}$ is extended by 0 to a smooth $L^{2}$ function on $M^{\prime}$, we get $h^{\prime}:=\left(h_{y} \rho_{y}\right) \cdot r^{*} e-h_{2} \in H^{2}\left(M^{\prime}, K_{L^{\prime}}\right)$. Finally, we set

$$
h:=\left.h^{\prime} \cdot\left(r^{*} f_{e} \cdot r^{*} e^{-1}\right)\right|_{M^{\prime}}
$$

Clearly, $h \in H^{2}\left(M^{\prime}\right)$ and it has a local peak point at $y$. Let us show that in fact $h$ has a peak point at $y$. This will complete the proof of the theorem.

Let $L_{1} \subset \subset L$ be a neighbourhood of $\bar{M}$. We set $L_{1}^{\prime}:=r^{-1}\left(L_{1}\right)$. Then by Proposition $\left.2.4 h_{1}\right|_{r^{-1}\left(L_{1} \backslash C_{M}\right)}$ is extended to a section of $K_{L_{1}^{\prime}}$ (denoted as before by $\left.h_{2}\right)$. By the definition, $h_{2}^{\prime}:=\left.h_{2} \cdot\left(r^{*} f_{e} \cdot r^{*} e^{-1}\right)\right|_{L_{1}^{\prime}}$ is a smooth holomorphic on $L_{1}^{\prime} \backslash S^{\prime}$ function. From the facts that $L_{1} \subset \subset L$, the $L^{2}$ norm $\|\cdot\|$ on $L_{1}^{\prime}$ is defined by a Riemannian volume form pulled back from $N$ using the meanvalue property for the plurisubharmonic function $\left|h_{2}^{\prime}\right|^{2}$ on $L_{1}^{\prime} \backslash S^{\prime}$ we get for some $c>0$, 


$$
\sup _{z \in M}\left\{\sum_{w \in r^{-1}(z), w \notin U_{y}}\left|h_{2}^{\prime}(w)\right|^{2}\right\} \leq c \cdot\left\|h_{2}^{\prime}\right\|^{2}<\infty .
$$

In addition, $h_{2}^{\prime}$ is continuous on $\bar{U}_{y} \cap \overline{M^{\prime}}$. These imply easily that $h$ is bounded outside $U \cap M^{\prime}$ for any neighbourhood $U$ of $y$ in $N^{\prime}$.

\section{References}

[Br1] A. Brudnyi, Representation of holomorphic functions on coverings of pseudoconvex domains in Stein manifolds via integral formulas on these domains, J. Funct. Anal. 231 (2006), no. 2, 418-437.

[Br2] Holomorphic functions of slow growth on coverings of pseudoconvex domains in Stein manifolds, Compos. Math. 142 (2006), no. 4, 1018-1038.

[Br3] , Solvable matrix representations of Kähler groups, Differential Geom. Appl. 19 (2003), no. 2, 167-191.

[C] H. Cartan, Sur les fonctions de plusieurs variables complexes: les espaces analytiques, in Proc. Internat. Congress Math. 1958, 33-52, Cambridge Univ. Press, New York.

[Ca] F. Campana, Ensembles de Green-Lazarsfeld et quotients résolubles des groupes de Kähler, J. Algebraic Geom. 10 (2001), no. 4, 599-622.

[D] J.-P. Demailly, Estimations $L^{2}$ pour l'opérateur $\bar{\partial}$ d'un fibré vectoriel holomorphe semi-positif au-dessus d'une variété kählérienne complète, Ann. Sci. École Norm. Sup. (4) 15 (1982), no. 3, 457-511.

[E] P. Eyssidieux, Sur la convexité holomorphe des revêtements linéaires réductifs d'une variété projective algébrique complexe, Invent. Math. 156 (2004), no. 3, 503-564.

[G] H. Grauert, On Levi's problem and the imbedding of real-analytic manifolds, Ann. of Math. (2) 68 (1958), 460-472.

[GHS] M. Gromov, G. Henkin and M. Shubin, Holomorphic $L^{2}$ functions on coverings of pseudoconvex manifolds, Geom. Funct. Anal. 8 (1998), no. 3, 552-585.

[GM] C. Grant and P. Milman, Metrics for singular analytic spaces, Pacific J. Math. 168 (1995), no. 1, 61-156.

[N] R. Narasimhan, Imbedding of holomorphically complete complex spaces, Amer. J. Math. 82 (1960), 917-934.

[O] T. Ohsawa, Complete Kähler manifolds and function theory of several complex variables. Sugaku Expositions 1 (1988), no. 1, 75-93.

[R] R. Remmert, Sur les espaces analytiques holomorphiquement séparables et holomorphiquement convexes, C. R. Acad. Sci. Paris 243 (1956), 118-121. 